\title{
Identification and characterization of neuroblasts in the subventricular zone and rostral migratory stream of the adult human brain
}

Congmin Wang ${ }^{1}$, Fang Liu ${ }^{1}$, Ying-Ying Liu ${ }^{2}$, Cai-Hong Zhao ${ }^{2}$, Yan You ${ }^{1}$, Lei Wang ${ }^{3}$, Jingxiao Zhang ${ }^{4}$, Bin Wei ${ }^{1}$, Tong Ma ${ }^{1}$, Qiangqiang Zhang ${ }^{1}$, Yue Zhang ${ }^{1}$, Rui Chen ${ }^{1}$, Hongjun Song ${ }^{5}$, Zhengang Yang ${ }^{1}$

${ }^{I}$ Institutes of Brain Science and State Key Laboratory of Medical Neurobiology, Fudan University, 138 Yixueyuan Road, Shanghai 200032, China; ${ }^{2}$ Institute of Neurosciences, The Fourth Military Medical University, Xi'an 710032, China; ${ }^{3}$ Department of Human Anatomy, Hebei Medical University, Shijiazhuang 050017, China; ${ }^{4}$ Department of Obstetrics and Gynecology, The Fourth Hospital of Shijiazhuang, Shijiazhuang 050011, China; ${ }^{5}$ Institute for Cell Engineering, Department of Neurology, Johns Hopkins University School of Medicine, Baltimore, MD 21205, USA

It is of great interest to identify new neurons in the adult human brain, but the persistence of neurogenesis in the subventricular zone (SVZ) and the existence of the rostral migratory stream (RMS)-like pathway in the adult human forebrain remain highly controversial. In the present study, we have described the general configuration of the RMS in adult monkey, fetal human and adult human brains. We provide evidence that neuroblasts exist continuously in the anterior ventral SVZ and RMS of the adult human brain. The neuroblasts appear singly or in pairs without forming chains; they exhibit migratory morphologies and co-express the immature neuronal markers doublecortin, polysialylated neural cell adhesion molecule and $\beta I I I-t u b u l i n$. Few of these neuroblasts appear to be actively proliferating in the anterior ventral SVZ but none in the RMS, indicating that neuroblasts distributed along the RMS are most likely derived from the ventral SVZ. Interestingly, no neuroblasts are found in the adult human olfactory bulb. Taken together, our data suggest that the SVZ maintains the ability to produce neuroblasts in the adult human brain.

Keywords: human; rhesus monkey; stem cells; neurogenesis; neuroblasts; subventricular zone; rostral migratory stream Cell Research (2011) 21:1534-1550. doi:10.1038/cr.2011.83; published online 17 May 2011

\section{Introduction}

Adult neural stem cells hold the potential for therapeutic applications and for mediating adaptation to normal and pathological stimuli. In most mammals, the generation of new neurons (neurogenesis) from neural stem cells persists throughout life in the subventricular zone (SVZ) of the lateral ventricle and in the subgranular zone of the dentate gyrus in the hippocampus [1-3].

In the adult mouse brain, SVZ neural stem cells (type $\mathrm{B}$ cells) generate rapidly dividing intermediate progeni-

Correspondence: Zhengang Yang

Tel: +86-21-5423-7872; Fax: +86-21-5423-7643

E-mail: yangz@fudan.edu.cn

Received 20 February 2011; revised 6 April 2011; accepted 18 April 2011; published online 17 May 2011 tor cells (type $\mathrm{C}$ cells), which in turn produce neuroblasts (type A cells) that migrate along the rostral migratory stream (RMS) into the olfactory bulb (OB). In the OB, they give rise to olfactory interneurons $[4,5]$. From the SVZ to the OB, the majority of migrating neuroblasts move along each other forming chains $[6,7]$.The RMS appears more prominent in the early postnatal rodent brain than in the adult rodent brain [8]. A similar migration has been described in the neonatal and adult monkey brain [9-13]. However, the general configuration of the RMS in coronal sections of the monkey brain has not yet been unequivocally shown. Moreover, the location where adult neuroblasts in the RMS traverse the monkey brain parenchyma before joining the olfactory tract (olfactory peduncle) remains largely unknown.

Accumulating evidence has demonstrated that SVZ neural stem cells (type B cells) are astrocyte-like, GFAP- 
expressing cells $[4,5]$. This characterization also includes humans, where SVZ astrocytes with the characteristics of neural stem cells were identified in vitro [14-16]. Previous studies have provided evidence suggesting that neurogenesis occurs in the adult human hippocampus [17]. Two studies have also indicated the presence of a putative RMS in the fetal human brain $[18,19]$. However, evidence for the presence of SVZ neurogenesis and an RMS-like pathway in the adult human brain remains highly controversial [14, 20-23].

In the present study, we have described the configuration of the RMS in adult monkey, fetal human and adult human brains. In the adult human brain, we find that there are neuroblasts in the anterior ventral SVZ and RMS, but not OB. Interestingly, these neuroblasts appear singly or in pairs without forming chains. Few of these neuroblasts appear to be actively proliferating in vivo. This work suggests that the generation of neuroblasts from neural stem and progenitor cells in the SVZ occurs in the adult human brain.

\section{Results}

The general configuration of the RMS in the adult monkey brain

Every day, new neurons (neuroblasts) are generated in the adult monkey SVZ, and they migrate through an RMS to the OB [9-13]. To characterize the distribution of neuroblasts in the SVZ and RMS of the adult rhesus monkey, we performed double immunostaining for doublecortin (Dcx) and polysialylated neural cell adhesion molecule (PSA-NCAM) in coronal brain sections. Neuroblasts in the monkey SVZ and RMS are Dcx+/PSA$\mathrm{NCAM}+$, and they form chain-like structures (Figure 1) [9-11, 13]. In the SVZ, neuroblasts are mainly found in the lateral and ventral regions (Figure 1D). Occasionally, $\mathrm{Dcx}+/ \mathrm{PSA}-\mathrm{NCAM}+$ cells are detected in the dorsal, medial and caudal SVZ, but they are sparse.

The shape of the RMS in the primate brain differs from that of rodents. As shown in Figure 1, the RMS in the adult monkey brain extends from the ventral horn of the lateral ventricle to the olfactory tract [9-13]. It is surprising that the anatomical location of the RMS in the adult monkey brain can be observed in a coronal brain section (Figure 1). As in rodents, the monkey RMS is mainly composed of migrating neuroblasts and astrocytes (Figure 1) [9, 10]. Indeed, SVZ neuroblasts in the floor of the anterior horn of the lateral ventricle start to form the RMS (Figure 1D). In the monkey forebrain, the RMS is located between the caudate nucleus and the rostrum of the corpus callosum (Figure 1D). Beneath the caudate nucleus, the RMS directly traverses the rostrum of the corpus callosum and takes a path from medial to lateral to enter the anterior olfactory nucleus (Figure 1D and 1I). In the anterior olfactory nucleus, neuroblasts appear to take a rostral turn and then migrate into the $\mathrm{OB}$ through the olfactory tract (Supplementary information, Figure S1). Some proliferating Dcx+ cells are present in the SVZ, as they express the cell proliferation markers MCM2 (Figure 1O-1S') and Ki67 [9]. Interestingly, in the adult monkey olfactory tract and $\mathrm{OB}$, nearly all $\mathrm{Dcx}+$ cells express PSA-NCAM and BIII-tubulin (Tuj1), but many PSA-NCAM+ and Tuj1+ cells do not express Dcx (Supplementary information, Figure S1).

\section{The RMS is prominent in the fetal human brain}

To identify and characterize neuroblasts in the human brain, we first tested the specificity of the antibodies used in this study by immunostaining human material. Previous studies have clearly shown that there is a novel population of Dcx + cells in the adult human temporal cortex [24]. Moreover, a fixation delay of up to $12 \mathrm{~h}$ has no significant difference in the pattern of Dcx immunoreactivity and the number of Dcx + cells in the brain section [24]. Consistent with this report, using both goat and rabbit polyclonal Dcx antibodies, we observed many Dcx+ cells in layer II of the inferior temporal gyrus of the adult human brain (Supplementary information, Figure S2A$\mathrm{S} 2 \mathrm{C})$. These Dcx + cells co-expressed PSA-NCAM and Tuj1 (Supplementary information, Figure S2B and S2C). We also evaluated the immunoreactivity of the MCM2 and Ki67 antibodies on cryostat sections of the human jejunum and glioblastoma multiforme (as a positive control from the central nervous system). The nuclei within the dividing cells of these tissues uniformly showed strong MCM2 and Ki67 double-labeling (Supplementary information, Figure S2D and S2E).

Having established that the antibodies are compatible with human tissues, we then used these hallmarks to identify neuroblasts in the human fetal and adult brain. In serial coronal sections of the fetal brain (2224 weeks of gestation), 4',6'-diamidino-2-phenylindole (DAPI) staining and double-labeling for Dcx and Tuj1 revealed that the RMS is prominent. The migratory stream characterized by high cell density (demonstrated by DAPI staining) was observed from the lateral SVZ to the olfactory tract and OB (Figure 2). As expected, a huge number of Dcx + cells were observed along the stream (Figure 2B-2J). These Dcx+ cells co-expressed Tuj1 and PSA-NCAM (Figure 2B-2J), suggesting that they are migrating neuroblasts. We also recognized that some neuroblasts formed chains within the RMS (Figure $2 \mathrm{E})$, much like chains of migrating neuroblasts in the SVZ and RMS of the rodent and monkey brain (Figure 1 

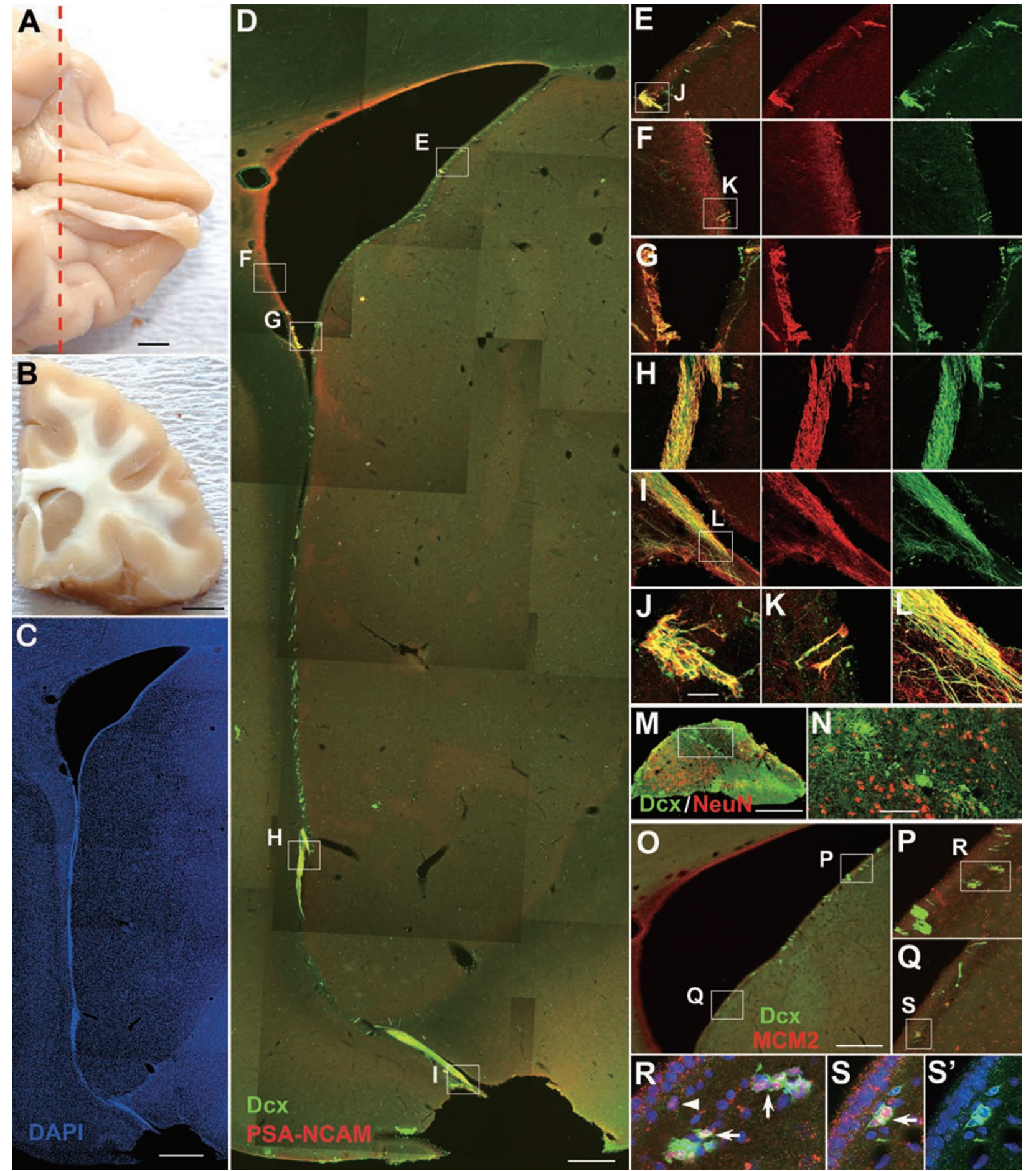

Figure 1 Neuroblasts in the SVZ and RMS of the adult rhesus monkey brain co-express Dcx and PSA-NCAM. (A) Photograph of the adult rhesus monkey brain (bottom view) showing the olfactory tract and OB. (B) The brain was cut coronally into 1.0-2.0 cm slabs. (C) DAPI staining of the coronal section shows the RMS. (D) The same section as C, double-immunostained for Dcx and PSA-NCAM. (E-I) Higher magnification of the boxed areas in D showing that the vast majority of Dcx+ cells express PSA-NCAM and vice versa. (J-L) Higher magnification of boxed areas in E, F and I. (M) Dcx/NeuN double immunostaining showing that Dcx+ cells migrate from the RMS to the anterior olfactory nucleus, which is located beneath (D). (N) Higher magnification of the boxed area in $\mathbf{M}$. (O) Dcx/MCM2 double immunostaining in the coronal sections of the adult monkey SVZ. (P, Q) Higher magnification of boxed areas in O. (R-S') Higher magnification of boxed areas in $\mathbf{P}$ and $\mathbf{Q}$. Note MCM2+/Dcx+ cells (arrows) and MCM2+ cells (arrowhead) in the SVZ. Scale bars represent $5 \mathrm{~mm}$ (A, B); $1 \mathrm{~mm}$ (C); $500 \mu \mathrm{m}$ (D, $\mathbf{M}$ and $\mathbf{0}$ ); $100 \mu \mathrm{m}$ (in $\mathbf{N}$ applies to $\mathbf{E}-\mathbf{I}, \mathbf{N}, \mathbf{P}$ and $\mathbf{Q}$ ) and $20 \mu \mathrm{m}$ (in $\mathbf{J}$ applies to $\mathbf{J}-\mathbf{L}$ and $\mathbf{R}-\mathbf{S}^{\prime}$ ). 

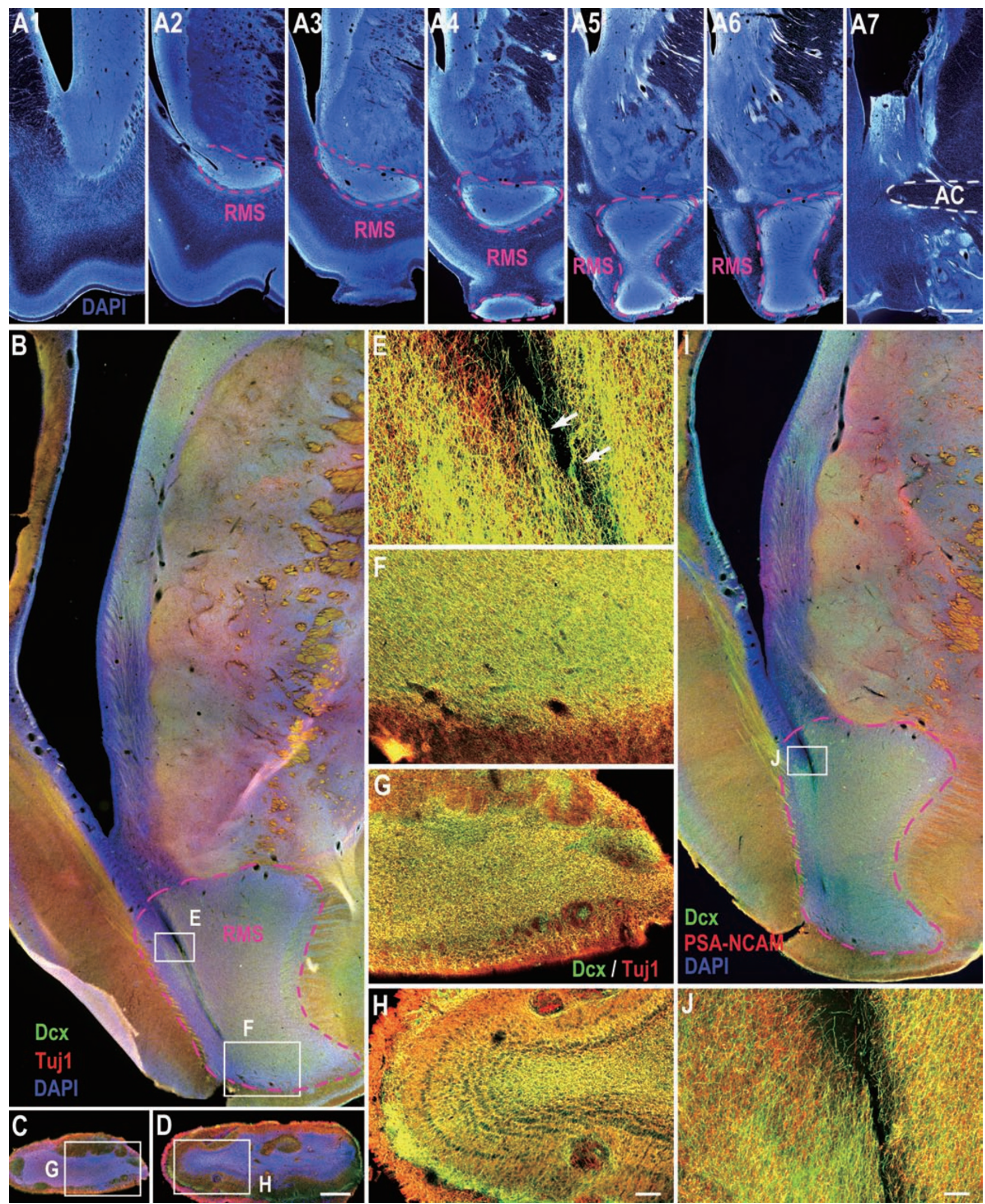
and Supplementary information, Figure S1) [6, 7, 9-11, 13]. Notably, at this time point, a ventral extension of the anterior horn of the lateral ventricle within the RMS was observed (Figure 2B and 2I). By contrast, the olfactory ventricle became closed as there was no open cavity in the olfactory tract and $\mathrm{OB}$ (Figure 2C and 2D) [25]. Taken together, these data suggest that the fetal human forebrain, similar to the rodent and monkey forebrain, harbors an SVZ-RMS-OB pathway through which newly born neuroblasts migrate from the SVZ into the OB.

\section{The RMS exists in the adult human brain}

To investigate whether an RMS-like pathway (the remnant of the fetal human RMS) exists in the adult human brain, we cut 160 serial $100-\mu \mathrm{m}$ coronal sections per hemisphere ( $n=2$, see Materials and Methods). Again, similar to what has been observed in rodents, monkeys and fetal humans, we identified an RMS-like pathway in the adult human brain parenchyma using DAPI staining, as cell density in the RMS is higher than that of the surrounding regions (Figure 3). This observation is further supported by GFAP immunostaining in $50-\mu \mathrm{m}$ coronal sections, as GFAP+ cells and their processes forming the glial tubes surround the RMS (Figures 3B', 3D', 3H', 4 and Supplementary information, Figure S4D-S4F). The configuration of the RMS in the adult human brain parenchyma demonstrated by DAPI staining in the present study was consistent with previous studies, in which the RMS was identified fortuitously using proliferating cell nuclear antigen (PCNA) immunostaining [21, 22].

Identification and characterization of neuroblasts in the adult human SVZ and RMS

Having identified that the RMS-like pathway may exist in the adult human brain, we then asked whether the SVZ and RMS contain migratory neuroblasts. Dcx, a microtubule-associated protein, which plays an important role in neuronal migration [26], is expressed by virtually all neuroblasts and immature neurons. A recent study reported the existence of Dcx $+/$ GFAP + mature astrocytes in the adult human cortex [27]; however, these results may depend on the type of antibody used [24]. In the present study, we clearly observed a population of Dcx + cells in the ventral SVZ and throughout the RMS using either immunofluorescence or immunoperoxidase staining (Figures 4-6, 8-9 and Supplementary information, Figures S3 and S4). However, we found no evidence of Dcx + cells in the dorsal, lateral, medial or caudal SVZ. GFAP/Dcx double immunostaining helped us to further identify Dcx+ cells in the RMS (Figure 4). These Dcx+ cells did not express GFAP (Figure 4B1-4E2). In the ventral SVZ, an average of $0.4 \mathrm{Dcx}+$ cells per section could be identified, whereas there were approximately 2.6 $\mathrm{Dcx}+$ cells in the RMS per section (totally analyzed 148 coronal sections that contain both the SVZ and RMS, Figures 4-6, 8-9 and Supplementary information, Figures S3 and S4). Thus, compared to the adult rodent and monkey brain, there are only a small number of Dcx + cells in the adult human SVZ and RMS.

To further characterize Dcx+ cells in the SVZ and RMS, Dcx/PSA-NCAM and Dcx/Tuj1 double immunostaining was performed. We found that all Dcx + cells in the SVZ and RMS expressed PSA-NCAM and Tuj1 (Figures 5 and 6). To the best of our knowledge, this is the first report showing Dcx+/PSA-NCAM+ and Dcx $+/$ Tuj1+ cells in the SVZ and RMS of the adult human brain. Interestingly, while PSA-NCAM+ cells and Tuj1+ cells were abundant in the SVZ, RMS, striatum and cortex, most of these cells, even those exhibiting a migratory morphology, did not express Dex (Figures 5 and 6, Supplementary information, Figures S2 and S4G-S4I). By contrast, in the adult rodent and monkey brain, except the monkey olfactory tract and OB (Supplementary information, Figure S1), the majority of Dcx+ cells in the SVZ and RMS expressed PSA-NCAM and vice versa (Figure 1). Thus, we propose that Dcx is a more reliable marker for neuroblasts in the adult human SVZ and RMS.

From the anterior ventral SVZ to the olfactory tract, the human RMS takes a caudal path. Therefore, the overall configuration of the human RMS cannot be shown in one coronal section (Figure 3). In all coronal sections examined, we did not observe any chains consisting of migrating neuroblasts in the SVZ or RMS. Instead, most $\mathrm{Dcx}+$ cells appeared as single cells and all these cells had the typical morphology of young migrating neuroblasts, i.e., an elongated morphology with unipolar or bipolar processes that are parallel to the direction of the

Figure 2 The RMS is prominent in the fetal human brain. (A1-A7) Representative coronal sections from the anterior SVZ to the anterior commissure (AC) stained for DAPI showing the RMS in the fetal human brain. Note that the intense DAPI staining, indicative of high cell density, distinguishes the SVZ and RMS from other regions of the forebrain. (B-D) Dcx/Tuj1 doubleimmunostained sections from the SVZ/RMS, olfactory tract and OB. (E-H) Higher magnification of the boxed areas in B-D. (I) Dcx/PSA-NCAM double immunostaining in the SVZ/RMS. (J) Higher magnification of the boxed area in I. Note that a lateral ventricular extension in B and I and chains of migrating neuroblasts (arrows in E) are seen in the RMS. Scale bars represent $1 \mathrm{~mm}$ (A7); $500 \mu \mathrm{m}$ (in D applies to B-D and I); $100 \mu \mathrm{m}$ (in $\mathbf{J}$ applies to $\mathbf{E}$ and $\mathbf{J}$ ); $50 \mu \mathrm{m}$ (in $\mathbf{H}$ applies to $\mathbf{F}-\mathbf{H}$ ). 

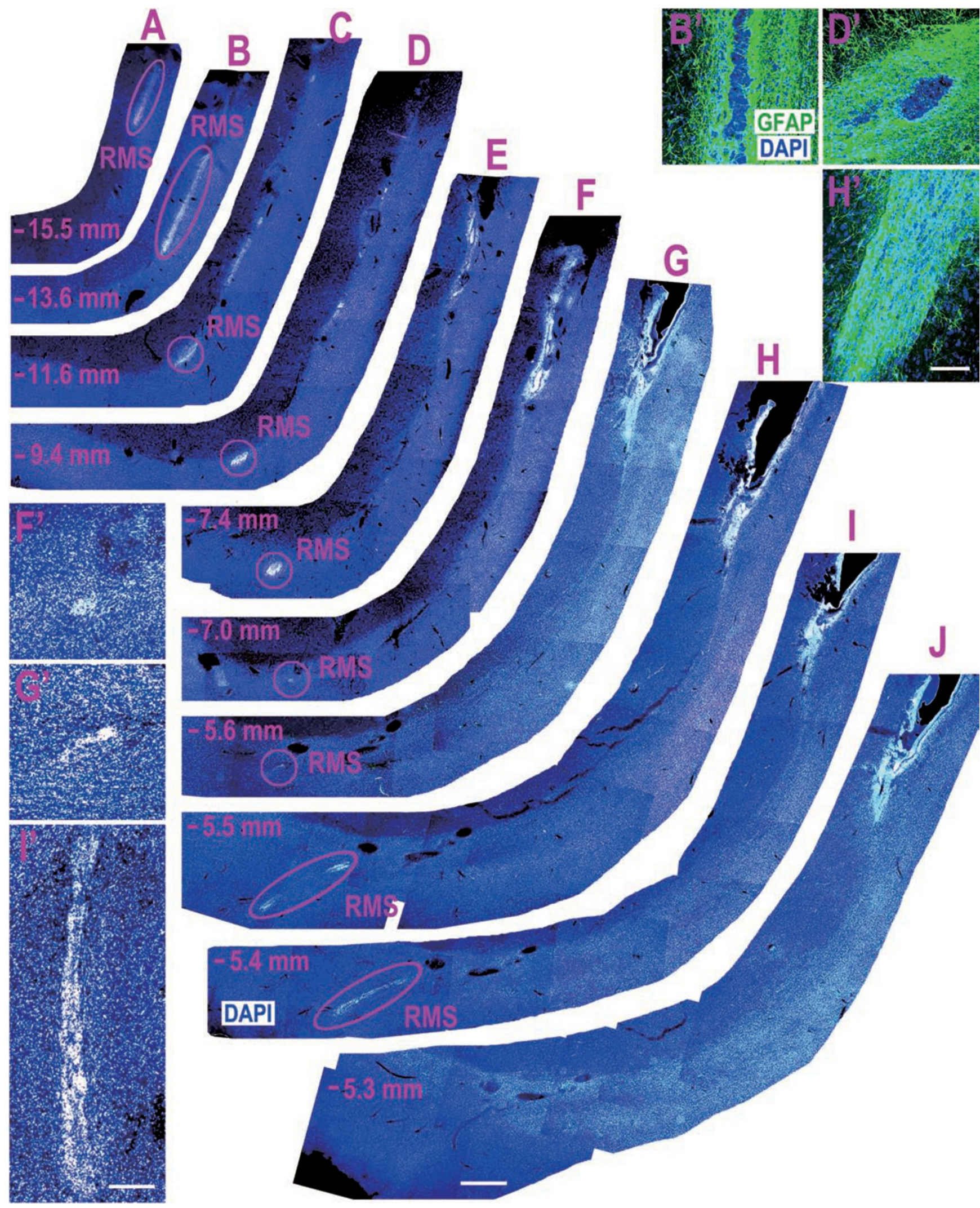

트
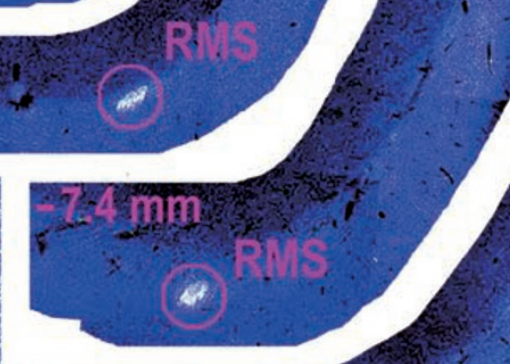

G

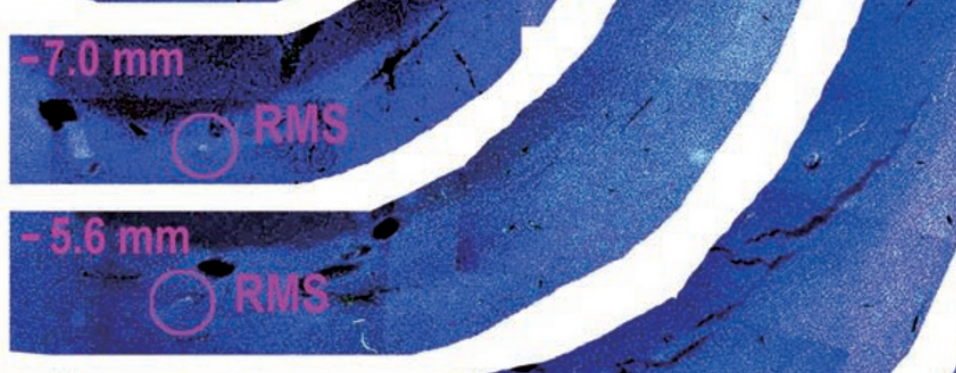

$\mathrm{H}$
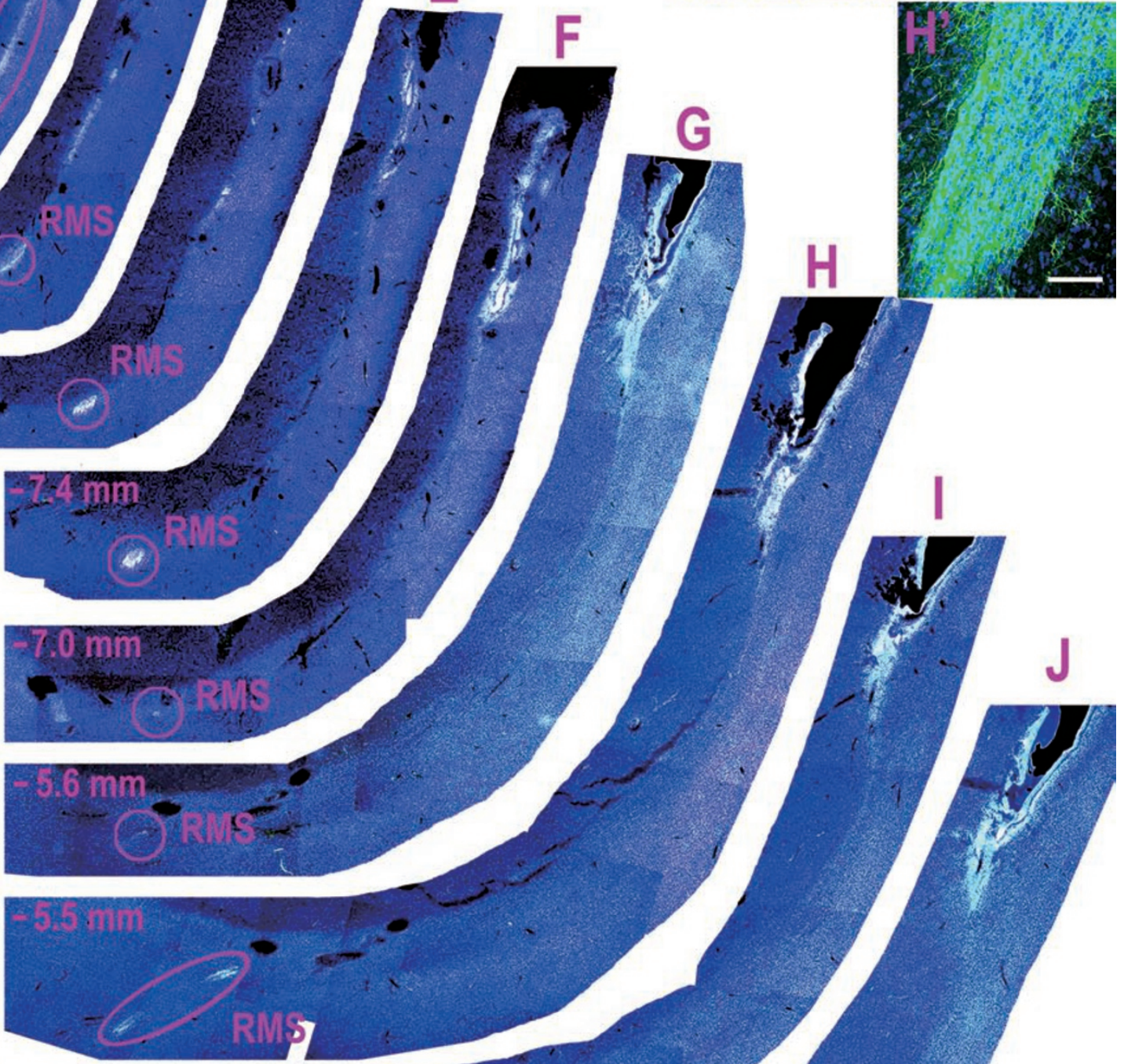

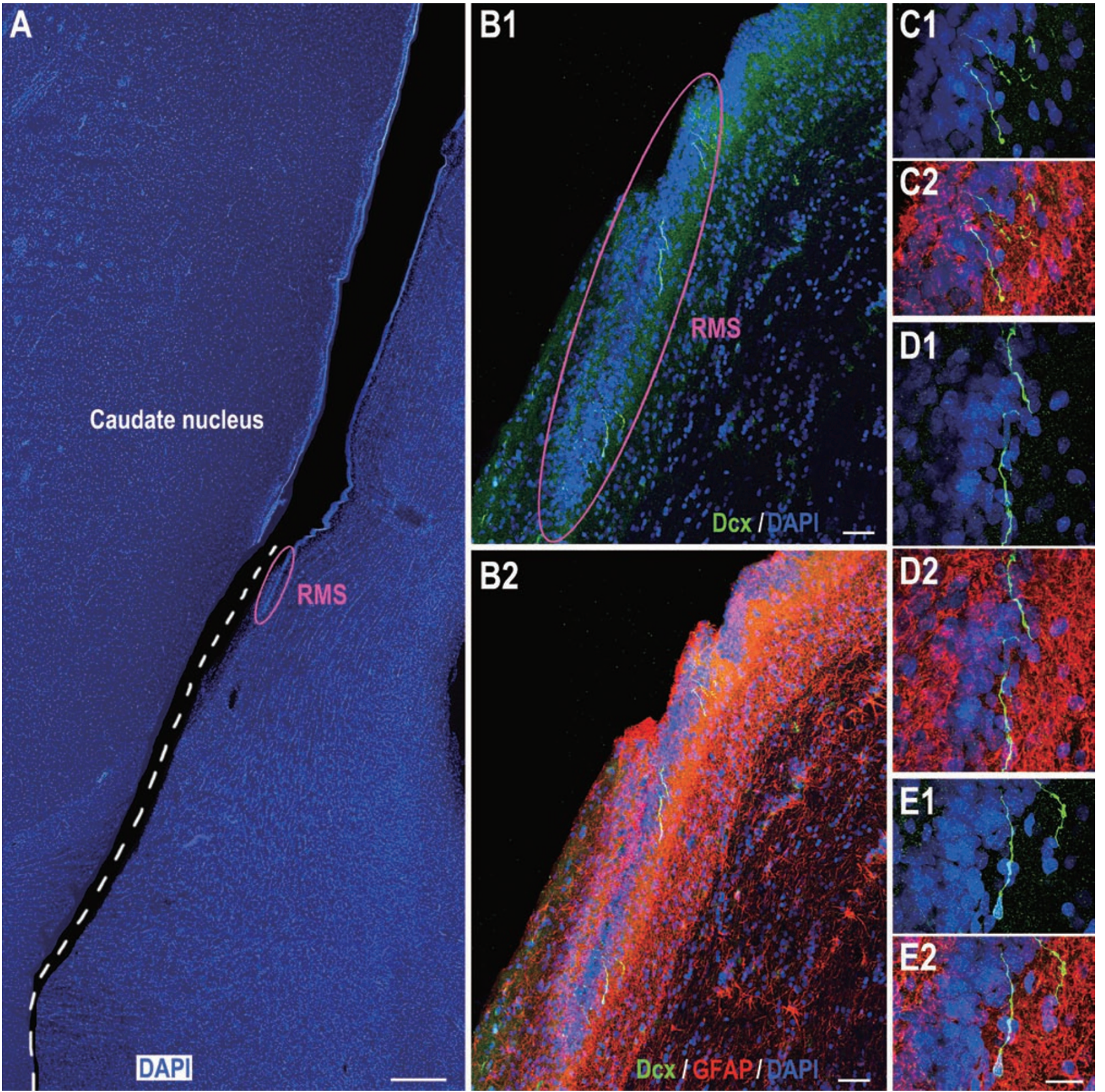

Figure 4 Dcx/GFAP double immunostaining of adult human brain sections indicating Dcx+ cells in the RMS. (A) One representative section stained for DAPI showing the ventral SVZ and RMS. The section broke at the ventral SVZ (dashed line) during processing. (B1, B2) The photomicrographs showing GFAP+ astrocytic cells surrounding individual Dcx+ cells in the RMS. (C1-E2) Higher magnification of Dcx+ cells in B1 and B2. Scale bars represent $1 \mathrm{~mm}$ (A); $100 \mu \mathrm{m}$ (B1, B2) and $20 \mu \mathrm{m}$ (in E2 applies to C1-E2).

Figure 3 The RMS-like pathway exists in the adult human forebrain. (A-J) Serial coronal sections stained for DAPI showing the RMS-like pathway in the adult human brain. Note that the RMS starts to appear at the ventral floor of the anterior horn, which is about $6.0 \mathrm{~mm}$ ahead of the ventral extension of the lateral ventricle that contains small canals formed by ependymal cells. (F', G', I') Higher magnification of the RMS in F, G, I. Note that the RMS in F is very thin. (B', D') GFAP expression in the RMS. The regions of GFAP and DAPI staining in B' and 'D' correspond to the same location of the RMS in B and D. Note the glial tube formed by GFAP+ cells. (H') GFAP immunostaining in the ventral floor of the lateral ventricle. Scale bars represent $1 \mathrm{~mm}$ (in J applies to A-J); $200 \mu \mathrm{m}$ (in l' applies to F', G' and I') and $100 \mu \mathrm{m}$ (in H' applies to B', D' and H'). 


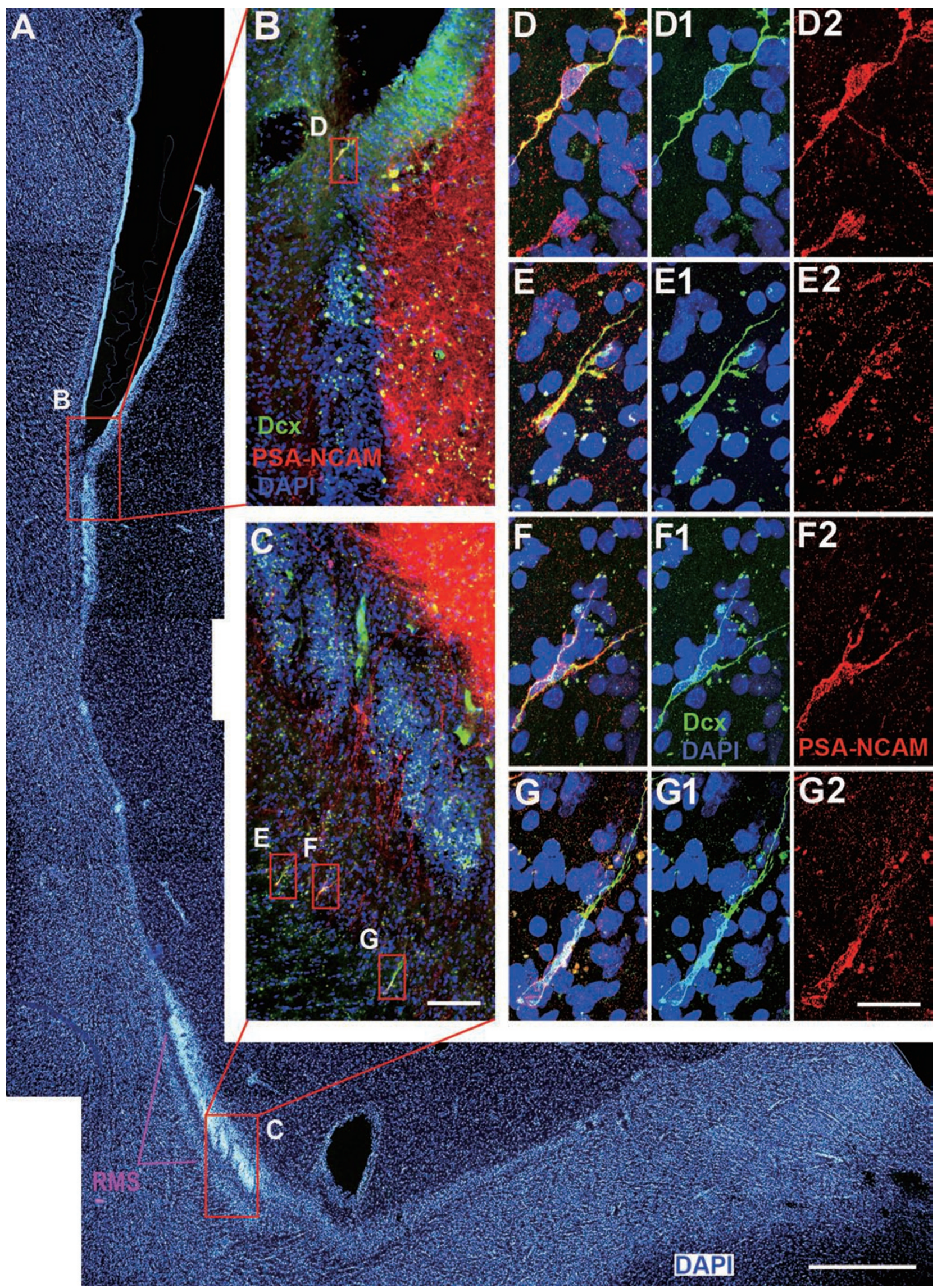


RMS (Figures 4-6, 8-9 and Supplementary information, Figures S3 and S4). The cell bodies of Dcx+ cells were $5 \mu \mathrm{m}$ on the shortest axis and $12 \mu \mathrm{m}$ on the longest axis. Interestingly, in the ventral SVZ, most, if not all, Dcx+ cells were located in the astrocyte ribbon, a band beneath the hypocellular gap in that cell bodies of astrocytes accumulate (Figures 6C, 6D and 8C) [14, 23]. There was also a subpopulation of $\mathrm{Dcx}+$ cells in the ventral $\mathrm{SVZ}$ and RMS that were frequently present as pairs of cell bodies closely associated with each other (16 of $63 \mathrm{Dcx}+$ cells) (Figures 5F, 6C, 8C and 8H). Moreover, the morphologies of Dcx + cells within the same pair were very similar (Figures 5F, 6C, 8C and 8H). Because there was only a small number of Dcx + cells in the SVZ and RMS, pre-embedding immuno-electron microscopy was used to identify these cells (Figure 6F-6I). Under the electron microscope, Dcx+ cells had an oval nucleus; Dcx protein appeared as clumps in the cytoplasm (Figure 6H). Many $\mathrm{Dcx}+$ processes were also observed (Figure 6I). These ultrastructural features bore a resemblance to the migrating neuroblasts (Type A cells) observed in mouse SVZ and RMS [6, 28].

While Dcx has been used in numerous studies as a hallmark for adult neurogenesis, the mere expression of Dcx in a small number of dispersed cells does not warrant the assumption that they are recently generated or migrating neuroblasts. Indeed, many species have Dcx+ cells in the adult brain, and these Dcx+ cells may be generated embryonically (Supplementary information, Figure S2) $[24,29,30]$. If Dcx+ cells in the adult human SVZ and RMS are bona fide young migrating neuroblasts, there should be at least a small number of Dcx+ cells capable of proliferating in vivo, which is typical of many neuroblasts in the adult rodent and monkey brain (Figure 1O-1S') [9, 10, 28, 31]. We first did an extensive analysis of the proliferating cells in the adult human SVZ and RMS. We found that there were an average of 1.8 MCM2+/Ki67+ cells in the ventral SVZ per section (65 cells from 37 sections); most MCM2+/Ki67+ cells were frequently present as pairs (56 of 65, Figure 7). Notably, all MCM2+ cells that appeared as pairs expressed Ki67 and vice versa (Figure 7B and 7E-7G). We then performed MCM2/Dcx double immunostaining. Detailed confocal Z-series analyses combined with 3-D reconstructions revealed that there was a very small subpopulation of Dcx+/MCM2+ cells (4 of $109 \mathrm{Dcx}+$ cells) in the anterior ventral-lateral SVZ (Figure 8A-8F). These
Dcx $+/ \mathrm{MCM} 2+$ cells were closely associated with each other and did not appear to be apoptotic-like cells as the characteristic condensed nuclei stained with DAPI were not seen (Figure 8D1-8F2). Interestingly, we found no evidence of proliferating Dcx + cells throughout the RMS (Figure 8G-8J). Actually, only 2 Ki67+/MCM2+ cells were found in the RMS from 37 sections, suggesting that the number of proliferating cells in the adult human RMS was much lower than that of the SVZ. Taken together, these results indicated that at least a very small number of Dcx + cells in the ventral SVZ are mitotically active neuroblasts and that other Dcx + cells that are continuously distributed in the SVZ and throughout the RMS are most likely immature postmitotic migrating neuroblasts. In support of this notion, we never observed Dcx + cells in the SVZ and RMS that express NeuN protein, a marker for mature neurons (Figure 9) [32].

\section{$D c x+$ cells are extremely rare in the adult human olfac- tory tract}

Because the RMS must enter the olfactory tract to reach the $\mathrm{OB}$, we asked whether the adult human olfactory tract and OB contain neuroblasts. To our surprise, we only detected extremely low numbers of convincing $\mathrm{Dcx}+/ \mathrm{PSA}-\mathrm{NCAM}+$ cells or processes in the olfactory tract (5 Dcx + cells in 81 sections, Figure 10). Moreover, we did not find Dcx+/PSA-NCAM+ or Dcx + cells in the OB (Supplementary information, Figure S5F and S5G). Similar to the adult monkey olfactory tract and OB, many PSA-NCAM+ and Tuj1+ cells were observed in those coronal and longitudinal sections of the olfactory tract and OB (Supplementary information, Figure S5). The vast majority of them, however, did not express Dcx (Supplementary information, Figure S5).

\section{Discussion}

The characteristics of neuroblasts in the adult monkey and fetal human SVZ-RMS-OB pathway are very similar to those of rodents. Indeed, these neuroblasts form chainlike structures to migrate into the $\mathrm{OB}$, and co-express Dcx, PSA-NCAM and Tuj1; some of them divide while migrating $[9-11,13,19]$. In the adult human brain, because there are only a small number of migratory neuroblasts in the SVZ and RMS, the neuroblasts cannot form chains. However, they are continuously distributed in the SVZ and RMS, possess the typical migratory morphol-

Figure 5 Neuroblasts in the adult human SVZ and RMS co-express Dcx and PSA-NCAM. (A) DAPI staining shows the anterior ventral SVZ and descending limb of the RMS. (B, C) Dcx+ cells express PSA-NCAM. Note that many PSA-NCAM+ cells in the SVZ, caudate nucleus and RMS do not express Dcx. (D-G) Higher magnification of boxed areas in B and C. Scale bars represent $1 \mathrm{~mm}$ (A); $100 \mu \mathrm{m}$ (in $\mathbf{C}$ applies to $\mathbf{B}, \mathbf{C}$ ) and $20 \mu \mathrm{m}$ (in $\mathbf{G} 2$ applies to D-G2). 

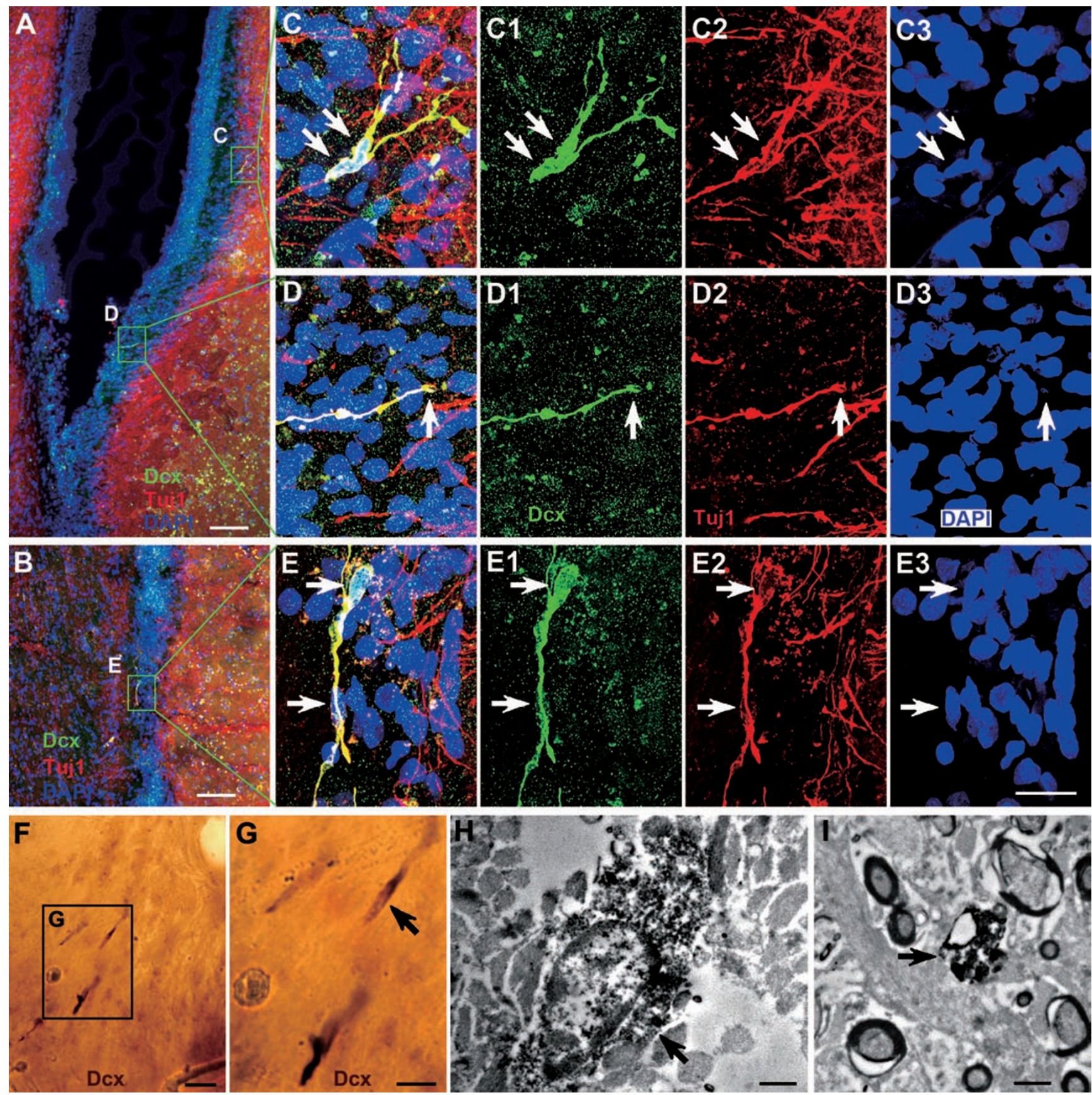

Figure 6 Neuroblasts in the adult human SVZ and RMS co-express Dcx and Tuj1. (A, B) Dcx+/Tuj1+ cells in the SVZ and RMS of the adult human brain. (C-E) Higher magnification of boxed areas in A, B. Note that many Tuj1+ cells were found in the SVZ, caudate nucleus and RMS, but they do not express Dcx. Arrows indicate neuroblast cell bodies or nuclei. (F, G) Dcx+ cells are identified by light microscopy. (H, I) One Dcx+ cell and process are identified by electron microscopy (arrows). Scale bars represent $100 \mu \mathrm{m}$ (A, B); $20 \mu \mathrm{m}$ (in E3 applies to C-E3 and F); $10 \mu \mathrm{m}$ (G) and $1 \mu \mathrm{m}$ (H, I).

ogy (i.e., an elongated shape with a small cell body) and co-express the immature neuronal markers Dcx, PSANCAM and Tuj1. Moreover, few of them are capable of proliferating in vivo. Because proliferating neuroblasts are only found in the ventral SVZ of the adult human brain, those neuroblasts that are continuously distributed along the RMS are most likely derived from the ventral SVZ. Interestingly, no neuroblasts are found in the adult human olfactory bulb. Taken together, our data suggest that the SVZ maintains the ability to produce neuroblasts 


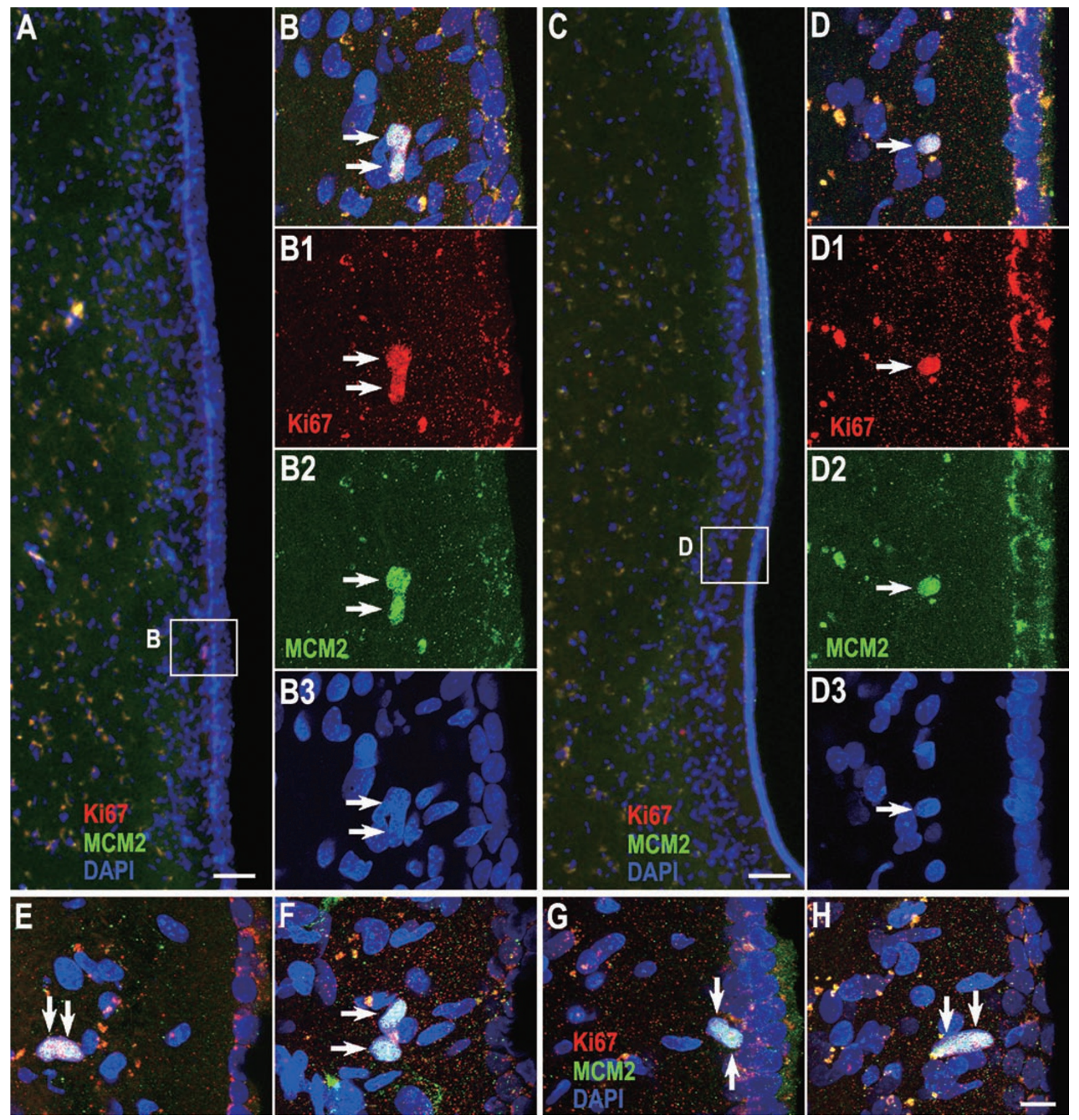

Figure 7 The ventral-lateral SVZ of adult human brains contains a few proliferative cells. (A, C) Representative sections double-immunostained for MCM2/Ki67. (B, D) Higher magnification of boxed areas in $\mathbf{A}$ and $\mathbf{C}$ showing MCM2+/Ki67+ cells (arrows). (E-H) The majority of MCM2+/Ki67+ cells appear in pairs. Scale bars represent $50 \mu \mathrm{m}$ (A, C); $10 \mu \mathrm{m}$ (in $\mathbf{H}$ applies to B-B3 and D-H).

in the adult human brain (Figure 11).

In the adult rodent and monkey brain, newly born neuroblasts in the SVZ migrate through the RMS to the OB $[6,7,9-13]$. However, whether there is an RMS- like pathway in the adult human brain remains highly controversial [14, 20-22]. In the present study, we first showed the configuration of the RMS in the adult rhesus monkey brain. Subsequently, we clearly showed that 

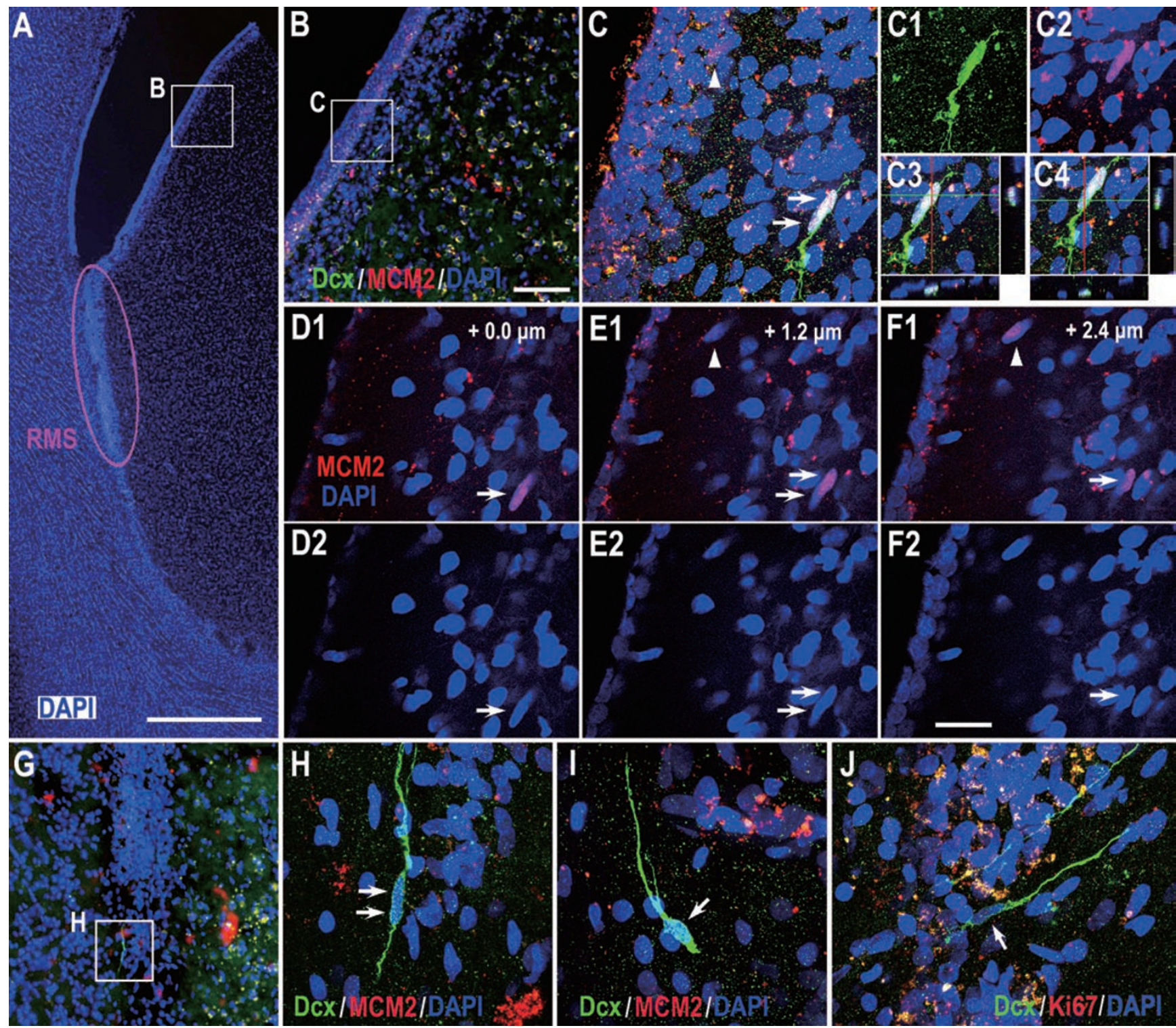

Figure $\mathbf{8}$ There is a very small number of MCM2+/Dcx+ cells in the ventral-lateral SVZ of the adult human brain. (A-C) MCM2/Dcx double-labeling combined with 3-D reconstructions showing 2 Dcx+ cells in the ventral-lateral SVZ expressing MCM2 (arrows). (D1-F2) Confocal Z sectioning was performed at 0.6- $\mu \mathrm{m}$ intervals using PlanApo 60× oil-immersion (NA = 1.42) objectives. Photomicrographs depict three confocal images in the Z-dimension showing two MCM2+/Dcx+ cells (arrows) and one MCM2+ cell (arrowhead). (G-J) Dcx+ cells do not express MCM2 or Ki67 in the RMS (arrows). Scale bars represent $1 \mathrm{~mm}$ (A); $100 \mu \mathrm{m}$ (in B applies to B and $\mathbf{G}$ ) and $20 \mu \mathrm{m}$ (in F2 applies to $\mathbf{C}-\mathbf{F} 2$ and $\mathbf{H}$-J).

the prominent RMS exists in the fetal human brain. Using DAPI staining combined with immunostaining for astrocytes and neuroblasts, we provided evidence for the possible presence of the RMS-like pathway in the adult human brain. Within this pathway, while neuroblasts do not form chains, they are present continuously. Interestingly, we found that some neuroblasts in the adult human SVZ and RMS frequently appeared in pairs. Like most single neuroblasts present in the SVZ and RMS, doublet neuroblasts also have an elongated shape with unipolar or bipolar processes, and their two-cell bodies are closely associated with each other. This unique feature of neuroblasts in the adult human brain has not been characterized in previous studies. Sanai et al. previously reported that the RMS-like pathway did not exist in the adult human brain [14]. The lack of chain migration could be the possible reason for missing the RMS, but these studies may also have unsuccessfully identified a correspond- 

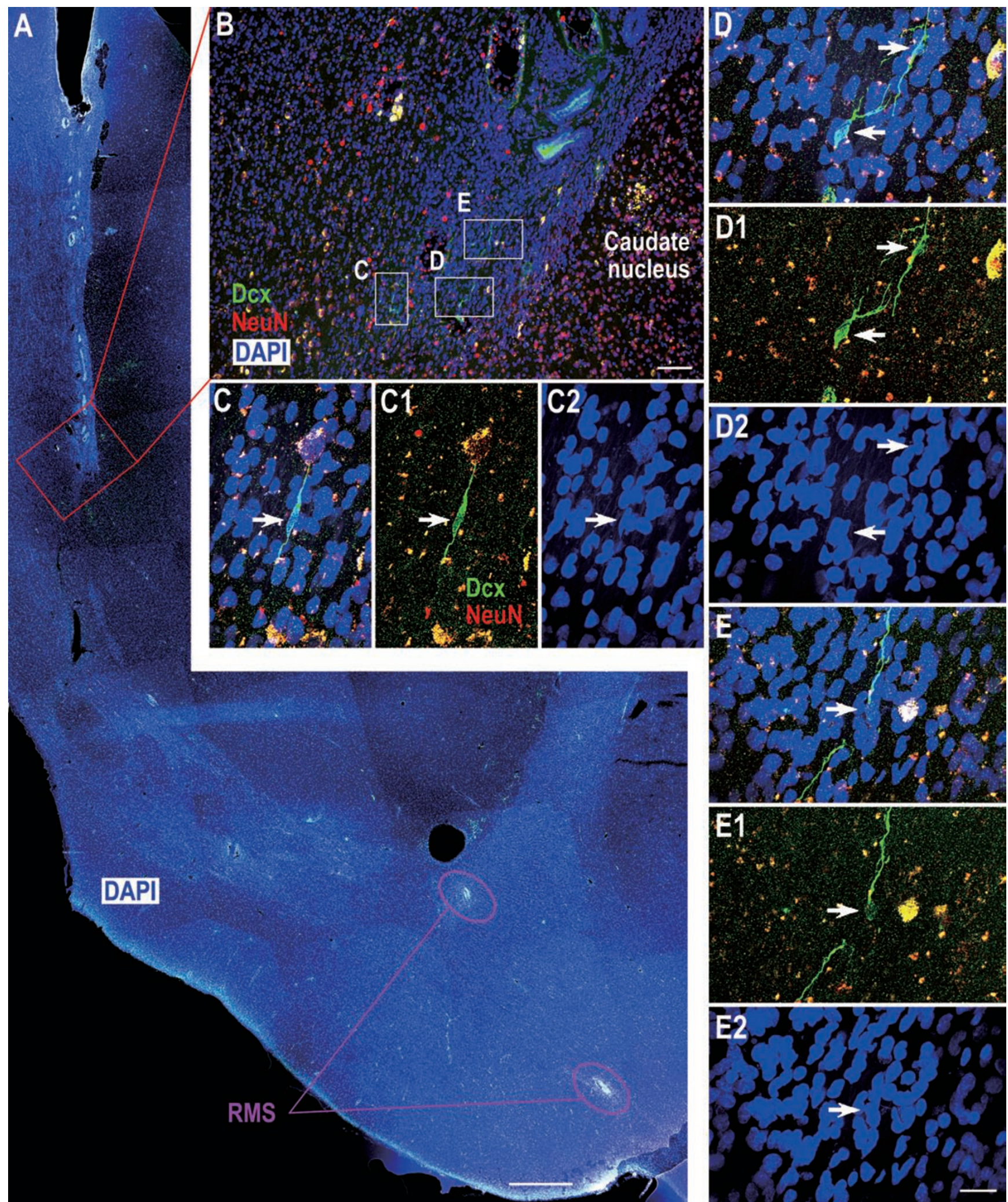

Figure $9 \mathrm{Dcx}+$ cells in the adult human SVZ and RMS are immature neuroblasts. (A) One representative section stained for DAPI showing the ventral extension of the lateral ventricle and RMS. Note that many ependymal cells form small canals in this region. (B) The photomicrograph showing Dcx/NeuN double immunostaining in the boxed area in A. (C-E2) Higher magnification of boxed areas in $\mathbf{B}$ showing that Dcx + cells do not express NeuN. Scale bars represent $1 \mathrm{~mm}(\mathbf{A}), 100 \mu \mathrm{m}(\mathbf{B})$ and $20 \mu \mathrm{m}$ (in E2 applies to C-E2). 

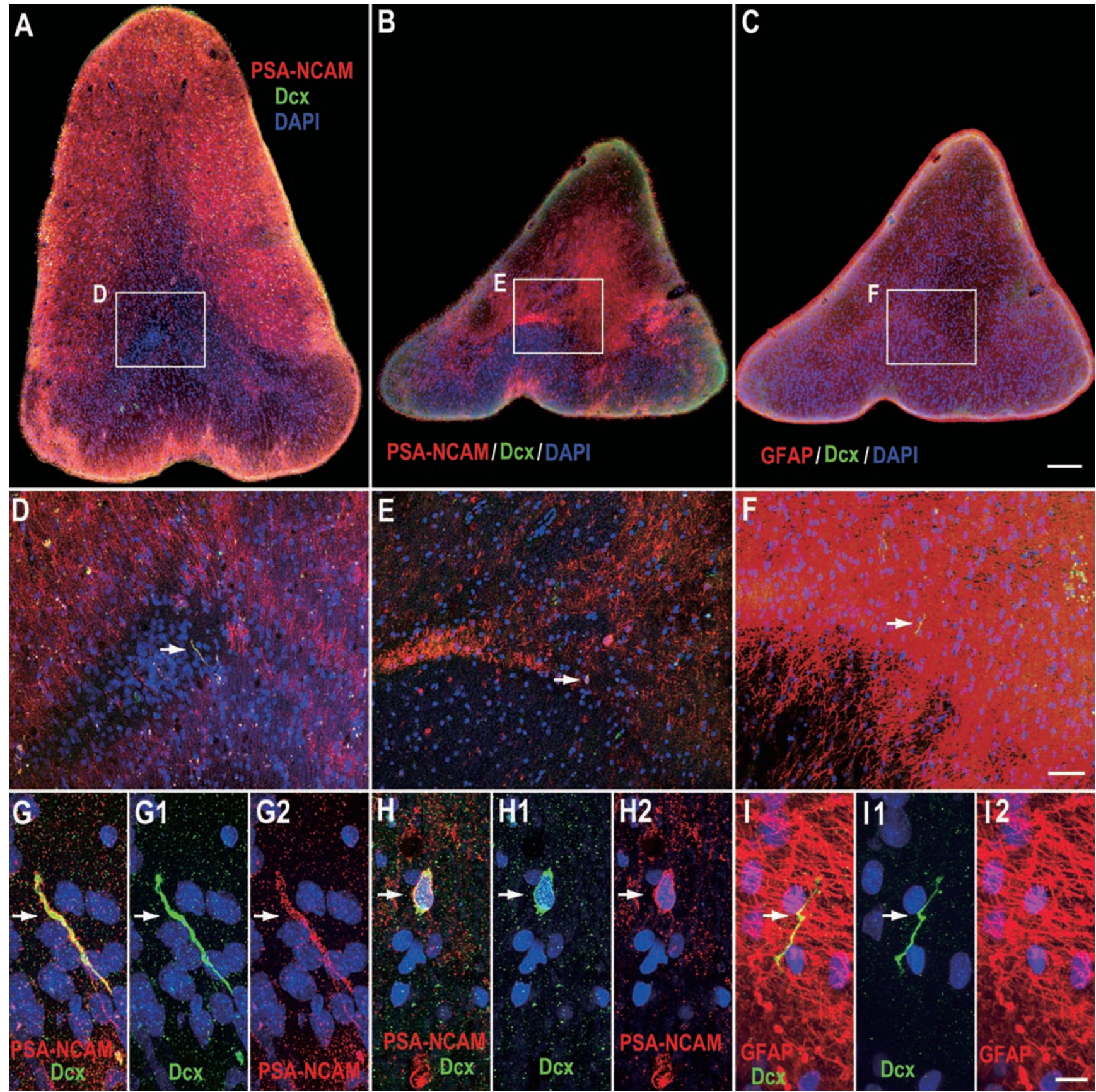

Figure $10 \mathrm{Dcx}+$ cells are extremely rare in the adult human olfactory tract. (A, B) Dcx/PSA-NCAM double immunostaining in coronal sections of the olfactory tract. (C) Dcx/GFAP double immunostaining in a coronal section of the olfactory tract. (D-F) Higher magnification of the boxed areas in A-C, respectively. (G-G2) Higher-magnification photomicrograph of 1 Dcx+/PSANCAM+ process (arrow). (H-H2) Higher-magnification photomicrograph of 1 Dcx+/PSA-NCAM+ cell (arrow). (I-I2) Highermagnification photomicrograph of $1 \mathrm{Dcx}+$ process (arrow). Note that the Dcx+ process does not express GFAP. Scale bars represent $200 \mu \mathrm{m}$ (in C applies to A-C); $50 \mu \mathrm{m}$ (in F applies to D-F) and $10 \mu \mathrm{m}$ (in $\mathbf{I 2}$ applies to $\mathbf{G}-\mathbf{I 2}$ ).

ingly small number of Dcx + cells that are present continuously in the SVZ and RMS of the adult human brain $[14,23]$.

Given that thousands of neuroblasts migrate into the
OB of adult rodents and monkeys and a population of neuroblasts is present in the adult human SVZ and RMS, one might speculate that there should be many migrating neuroblasts in the adult human OB. However, in the 


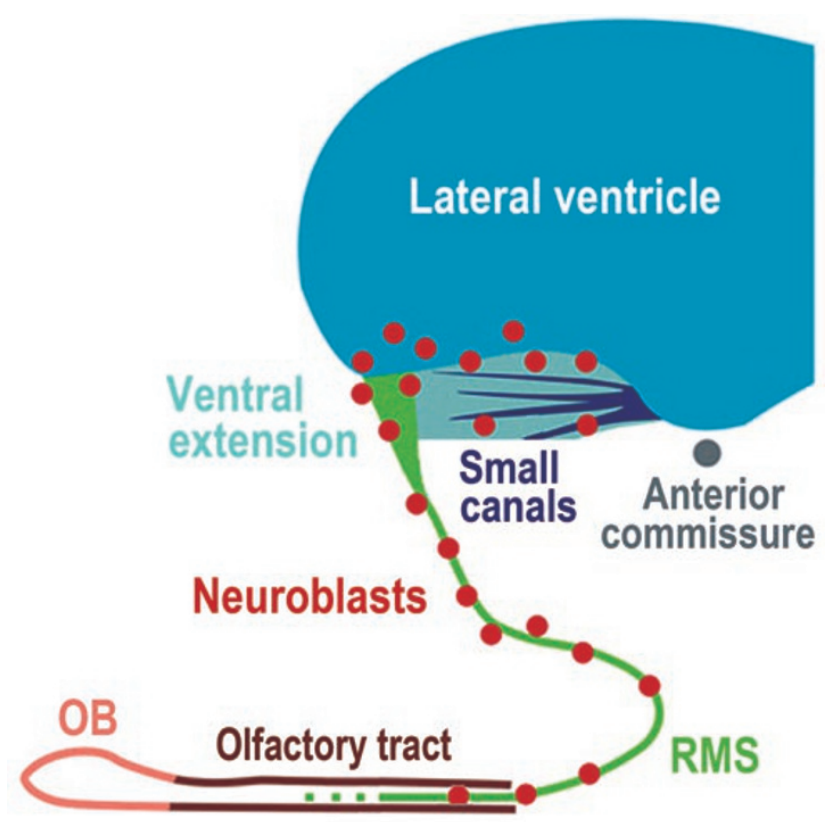

Figure 11 Schematic depicting neuroblasts in the SVZ and RMS. While neuroblasts exist continuously in the adult human ventral SVZ and RMS, proliferating neuroblasts are only found in the ventral SVZ, indicating that the SVZ maintains the ability to produce neuroblasts in the adult human brain.

adult human olfactory tract, only very few Dcx+/PSA$\mathrm{NCAM}+$ cells and processes were detected, suggesting that migrating neuroblasts are extremely rare in this region. In contrast to previous studies [33], we also found no evidence of Dcx+/Tuj1+ or Dcx + cells in the adult human OB. Recent work has shown that neuroblasts clear the path of glial processes for their migration in the RMS of the adult mouse brain [34]. Given that neuroblasts actively control the formation and maintenance of their own migratory route [34], it is difficult to imagine that such a small number of neuroblasts can successfully establish a long and complex migratory route from the $\mathrm{SVZ}$ to the OB. Thus, it is likely that migration from the adult human SVZ to the OB is an impossible mission for neuroblasts. This also raises fundamental questions about the role and final fate of neuroblasts in the SVZ and RMS in the adult human brain. However, we cannot exclude the possibility that young neuroblasts in the adult human SVZ and RMS stop expressing Dcx once they reach the OB.

Curtis et al. previously established a similar RMSlike pathway from the adult human SVZ to the OB using PCNA, PSA-NCAM and Tuj1 immunostaining [21]. The general configuration of the RMS in the adult human brain parenchyma demonstrated by DAPI staining in the present study is consistent with these studies [21,
22]. However, it is worth noting that the large number of PCNA+ cells in the RMS shown in these studies [21, 22] does not necessarily mean that they are proliferating cells. Indeed, we and others only found a very small number of MCM2+ and Ki67+ cells in the SVZ and RMS of the adult human brain (Figures 7 and 8) [14, 20, 23]. Massive PSA-NCAM+ and Tuj1+ cells do not guarantee that they are real migrating neuroblasts in the adult human brain because they neither form chains nor have the typical migratory morphology [21]. Moreover, it is unclear whether these cells express Dcx. In addition, the persistent ventricular lumen connecting the human SVZ to the OB reported by previous studies [21, 22] was never observed in the six samples examined in this study.

A subpopulation of GFAP+ cells in a unique SVZ astrocyte ribbon of the adult human brain has been identified as neural stem cells in vitro [14-16]. Some proliferating GFAP+ cells in the anterior ventral-lateral SVZ have also been observed $[14,23]$. In this region, we also observed a small number of MCM2+/Ki67+ cells. Moreover, very few mitotically active Dcx + cells were detected, suggesting that some SVZ neuroblasts are newly generated. Interestingly, we also found that neuroblasts in the adult human SVZ and RMS frequently appeared as doublets but not triplets, suggesting that neural progenitor cells divide symmetrically to produce two neuroblasts in the adult human brain.

In conclusion, we provide compelling evidence that new neuroblasts are continuously produced in the SVZ of the adult human brain. The largest germinal region in the adult brain is the SVZ, which shares many common features in most mammals. Thus, neural stem cells and newly born neuroblasts in the adult human SVZ might be used for neuroprotective and neuroregenerative therapy in the future, as many promising studies conducted in rodent brains have been reported.

\section{Materials and Methods}

\section{Animal tissue preparation}

All animal care and experiments were conducted in accordance with institutional guidelines. Two adult (6 and 7 years of age) rhesus monkeys were deeply anesthetized and then perfused with PBS followed by $4 \%$ paraformaldehyde (PFA). The brains were removed and postfixed with 4\% PFA for $48 \mathrm{~h}$. The brains were then cut coronally into approximately $1.0-2.0 \mathrm{~cm}$ slabs and cryoprotected in $30 \%$ sucrose in $0.1 \mathrm{M}$ sodium phosphate buffer at $4{ }^{\circ} \mathrm{C}$ for $72 \mathrm{~h}$. The brain tissue samples were frozen in embedding medium (O.C.T., Sakura Finetek) on dry ice/ethanol slush.

\section{Human tissue collection}

The collection of human tissue was in accordance with institutional guidelines and the study design was approved by the institutional review board (Ethics Committee) of Fudan University 
Shanghai Medical School.

Two fetal human brains (22-24 weeks of gestation) were obtained at autopsy within $3 \mathrm{~h}$ of spontaneous abortion. Brains were examined only with the informed consent of the patients. Brain tissue was fixed in $4 \%$ PFA at $4{ }^{\circ} \mathrm{C}$ for 1 week and then cryoprotected in $30 \%$ sucrose for $72 \mathrm{~h}$ at $4{ }^{\circ} \mathrm{C}$. The brain tissue samples were frozen in OCT on dry ice/ethanol slush.

Six adult human brains were obtained at autopsy from subjects of different ages (between 35 and 70 years), with no history of neuropathology. The average postmortem delay was $16 \mathrm{~h}$. Some specimens were obtained as early as 3-6 h postmortem. Brains were either removed at autopsy and suspended in 4\% PFA for $24 \mathrm{~h}$ or fixed by bilateral perfusion with $4 \%$ PFA for $1-2 \mathrm{~h}$ through the internal carotid arteries. The brains were then cut into $2.0-4.0-\mathrm{cm}-$ thick slabs. The slabs were postfixed in $4 \%$ PFA for 1 week and then cryoprotected in $30 \%$ sucrose for $72 \mathrm{~h}$ at $4{ }^{\circ} \mathrm{C}$. The brain tissue samples were frozen in OCT on dry ice/ethanol slush.

Three glioblastoma tissues were collected during neurosurgical procedures. Two human jejunal segments were collected from patients undergoing surgery for gastric bypass. These samples were used as positive controls in MCM2 and Ki67 immunohistochemical staining.

\section{Immunohistochemistry}

Immunohistochemical staining was performed on $60 \mu \mathrm{m}$ (fetal human brains) or $50 \mu \mathrm{m}$ (adult monkey and human brain) freefloating serial coronal sections in 12- or 24-well cell culture plates. Sections were blocked for $1-3 \mathrm{~h}$ in TBS with $1 \%$ Triton X-100 and $10 \%$ donkey serum. All primary antibodies were incubated for $48 \mathrm{~h}$ at $4{ }^{\circ} \mathrm{C}$. We used the following primary antibodies: goat antiDcx (1:100, Santa Cruz, sc-8066), rabbit anti-Dcx (1:500, Abcam, ab-18723), rabbit anti-GFAP (1:500, Dako, Z0334), rabbit antiKi67 (1:500, Vector Laboratories, VP-K451), Goat anti-MCM2 (1:300, Santa Cruz, sc-9839), mouse anti-NeuN (1:500, Millipore, MAB377), mouse anti-PSA-NCAM (1:600, Millipore, MAB5324) and mouse anti-Tuj1 (1:500, Covance, MMS-435P).

Secondary antibodies against the appropriate species were incubated for 3-5 h at room temperature (all from Jackson, 1:200). Fluorescently stained sections were then washed, counterstained with DAPI (Sigma, $200 \mathrm{ng} / \mathrm{ml}$ ) for $5 \mathrm{~min}$, and coverslipped with Gel/Mount (Biomeda, Foster City, CA, USA). Streptavidin and diaminobenzidine (DAB) were used to visualize the reaction product for bright-field staining sections. Omission of primary antibodies eliminated the staining.

To study the overall configuration of the RMS in the adult human brain, two right hemispheres were cut into about 160 serial $100-\mu \mathrm{m}$ coronal sections from the rostral SVZ to the anterior commissure $(-16.0$ to $0.0 \mathrm{~mm}$ from the vertical line that passes through the center of the anterior commissure) [35]. All of these sections were only counterstained with DAPI.

\section{Microscopy}

Fluorescently immunostained sections were analyzed on an Olympus FV1000 confocal laser scanning microscope. Confocal Z sectioning was performed using a $20 \times(\mathrm{NA}=0.75)$ or $60 \times(\mathrm{NA}=$ 1.42) objective for single, double and triple labeling. The Kalman filter mode was used during scanning. Images were acquired and a Z-stack was reconstructed using the Olympus FV10-ASW software, cropped, adjusted and optimized in Photoshop 9.0. Images of enzyme histochemistry-labeled sections and some fluorescently immunolabeled sections were acquired using an Olympus BX 51 microscope.

\section{Electron microscopy}

For Dcx pre-embedding immunostaining, $50 \mu \mathrm{m}$ sections from adult human brains were incubated for $48 \mathrm{~h}$ at $4{ }^{\circ} \mathrm{C}$ with anti-Dcx (1:100, Santa Cruz, sc-8066). The second antibody (biotinylated donkey-anti-goat) was incubated for $4 \mathrm{~h}$ at room temperature. Sections were then incubated with streptavidin-coupled HRP for $2 \mathrm{~h}$ and revealed with DAB. The sections after immunostaining were post-fixed in $2 \%$ osmium for $2 \mathrm{~h}$, rinsed, dehydrated and embedded in Araldite. Dcx + cells in the SVZ and RMS were first identified by light microscopy. To further characterize Dcx + cells, we cut $60 \mathrm{~nm}$ ultrathin sections with a diamond knife. The sections were stained with lead citrate and uranyl acetate, and examined under a Philips CM120 electron microscope.

\section{Acknowledgment}

This work was supported by the National Natural Science Foundation of China (30870804, 30900425, 30970949, 30990261, 30821002 and 31028009), Shanghai Shu-Guang project (09SG05) and National Basic Research Program of China (2010CB945500, 2011CB504400). We thank the staff at Chinese Brain Bank Center, Wuhan, China and Red Cross Society of China, Shanghai Branch at the Fudan University for providing access to donated human brains. We thank Dr John Rubinstein (University of California, San Francisco, USA) for his helpful comments on the manuscript.

\section{References}

1 Ming GL, Song H. Adult neurogenesis in the mammalian central nervous system. Annu Rev Neurosci 2005; 28:223-250.

2 Kriegstein A, Alvarez-Buylla A. The glial nature of embryonic and adult neural stem cells. Annu Rev Neurosci 2009; 32:149-184.

3 Suh H, Deng W, Gage FH. Signaling in adult neurogenesis. Annu Rev Cell Dev Biol 2009; 25:253-275.

4 Doetsch F, Caille I, Lim DA, Garcia-Verdugo JM, AlvarezBuylla A. Subventricular zone astrocytes are neural stem cells in the adult mammalian brain. Cell 1999; 97:703-716.

5 Mirzadeh Z, Merkle FT, Soriano-Navarro M, Garcia-Verdugo JM, Alvarez-Buylla A. Neural stem cells confer unique pinwheel architecture to the ventricular surface in neurogenic regions of the adult brain. Cell Stem Cell 2008; 3:265-278.

6 Lois C, Garcia-Verdugo JM, Alvarez-Buylla A. Chain migration of neuronal precursors. Science 1996; 271:978-981.

7 Doetsch F, Alvarez-Buylla A. Network of tangential pathways for neuronal migration in adult mammalian brain. Proc Natl Acad Sci USA 1996; 93:14895-14900.

8 Luskin MB. Restricted proliferation and migration of postnatally generated neurons derived from the forebrain subventricular zone. Neuron 1993; 11:173-189.

9 Gil-Perotin S, Duran-Moreno M, Belzunegui S, Luquin MR, Garcia-Verdugo JM. Ultrastructure of the subventricular zone in Macaca fascicularis and evidence of a mouse-like migratory stream. J Comp Neurol 2009; 514:533-554.

10 Pencea V, Bingaman KD, Freedman LJ, Luskin MB. Neu- 
rogenesis in the subventricular zone and rostral migratory stream of the neonatal and adult primate forebrain. Exp Neurol 2001; 172:1-16.

11 Kornack DR, Rakic P. The generation, migration, and differentiation of olfactory neurons in the adult primate brain. Proc Natl Acad Sci USA 2001; 98:4752-4757.

12 Bedard A, Levesque M, Bernier PJ, Parent A. The rostral migratory stream in adult squirrel monkeys: contribution of new neurons to the olfactory tubercle and involvement of the antiapoptotic protein Bcl-2. Eur J Neurosci 2002; 16:1917-1924.

13 Sawamoto K, Hirota Y, Alfaro-Cervello C, et al. Cellular composition and organization of the subventricular zone and rostral migratory stream in the adult and neonatal common marmoset brain. J Comp Neurol 2011; 519:690-713.

14 Sanai N, Tramontin AD, Quinones-Hinojosa A, et al. Unique astrocyte ribbon in adult human brain contains neural stem cells but lacks chain migration. Nature 2004; 427:740-744.

15 Leonard BW, Mastroeni D, Grover A, et al. Subventricular zone neural progenitors from rapid brain autopsies of elderly subjects with and without neurodegenerative disease. J Comp Neurol 2009; 515:269-294.

16 van den Berge SA, Middeldorp J, Zhang CE, et al. Longterm quiescent cells in the aged human subventricular neurogenic system specifically express GFAP-delta. Aging Cell 2010; 9:313-326.

17 Eriksson PS, Perfilieva E, Bjork-Eriksson T, et al. Neurogenesis in the adult human hippocampus. Nat Med 1998; 4:13131317.

18 Weickert CS, Webster MJ, Colvin SM, et al. Localization of epidermal growth factor receptors and putative neuroblasts in human subependymal zone. J Comp Neurol 2000; 423:359372.

19 Guerrero-Cazares H, Gonzalez-Perez O, Soriano-Navarro M, et al. Cytoarchitecture of the lateral ganglionic eminence and rostral extension of the lateral ventricle in the human fetal brain. J Comp Neurol 2011; 519:1165-1180.

20 Sanai N, Berger MS, Garcia-Verdugo JM, Alvarez-Buylla A. Comment on "Human neuroblasts migrate to the olfactory bulb via a lateral ventricular extension". Science 2007; 318:393.

21 Curtis MA, Kam M, Nannmark U, et al. Human neuroblasts migrate to the olfactory bulb via a lateral ventricular extension. Science 2007; 315:1243-1249.

22 Kam M, Curtis MA, McGlashan SR, et al. The cellular com- position and morphological organization of the rostral migratory stream in the adult human brain. $J$ Chem Neuroanat 2009; 37:196-205.

23 Quinones-Hinojosa A, Sanai N, Soriano-Navarro M, et al. Cellular composition and cytoarchitecture of the adult human subventricular zone: a niche of neural stem cells. J Comp Neurol 2006; 494:415-434.

24 Liu YW, Curtis MA, Gibbons HM, et al. Doublecortin expression in the normal and epileptic adult human brain. Eur $J$ Neurosci 2008; 28:2254-2265.

25 Humphrey TJ. The development of the olfactory and the accessory olfactory formations in human embryos and fetuses. $J$ Comp Neurol 1940; 73:431-468.

26 Gleeson JG, Lin PT, Flanagan LA, Walsh CA. Doublecortin is a microtubule-associated protein and is expressed widely by migrating neurons. Neuron 1999; 23:257-271.

27 Verwer RW, Sluiter AA, Balesar RA, et al. Mature astrocytes in the adult human neocortex express the early neuronal marker doublecortin. Brain 2007; 130:3321-3335.

28 Doetsch F, Garcia-Verdugo JM, Alvarez-Buylla A. Cellular composition and three-dimensional organization of the subventricular germinal zone in the adult mammalian brain. $J$ Neurosci 1997; 17:5046-5061.

29 Zhang XM, Cai Y, Chu Y, et al. Doublecortin-expressing cells persist in the associative cerebral cortex and amygdala in aged nonhuman primates. Front Neuroanat 2009; 3:17.

30 Gomez-Climent MA, Castillo-Gomez E, Varea E, et al. A population of prenatally generated cells in the rat paleocortex maintains an immature neuronal phenotype into adulthood. Cereb Cortex 2008; 18:2229-2240.

31 Menezes JR, Smith CM, Nelson KC, Luskin MB. The division of neuronal progenitor cells during migration in the neonatal mammalian forebrain. Mol Cell Neurosci 1995; 6:496508.

32 Mullen RJ, Buck CR, Smith AM. NeuN, a neuronal specific nuclear protein in vertebrates. Development 1992; 116:201211.

33 Bedard A, Parent A. Evidence of newly generated neurons in the human olfactory bulb. Brain Res 2004; 151:159-168.

34 Kaneko N, Marin O, Koike M, et al. New neurons clear the path of astrocytic processes for their rapid migration in the adult brain. Neuron 2010; 67:213-223.

35 Mai JK, Paxinos G, Voss T. Atlas of the Human Brain. 3rd Edition. San Diego: Academic Press, 2008.

(Supplementary information is linked to the online version of the paper on the Cell Research website.) 